\title{
L'expérience du courriel en situation professionnelle : représentations de l'activité, jugements et affects
}

The experience of email at work: Representations, judgments and affects

\section{Nadia Gauducheau}

\section{(2) OpenEdition}

Journals

Édition électronique

URL : http://journals.openedition.org/activites/345

DOI : 10.4000/activites.345

ISSN : 1765-2723

Éditeur

ARPACT - Association Recherches et Pratiques sur les ACTivités

\section{Référence électronique}

Nadia Gauducheau, «L'expérience du courriel en situation professionnelle : représentations de l'activité, jugements et affects », Activités [En ligne], 9-2 I Octobre 2012, mis en ligne le 15 octobre 2012, consulté le 01 mai 2019. URL : http://journals.openedition.org/activites/345 ; DOI : 10.4000/ activites.345

\section{(c) (i) 9}

Activités est mis à disposition selon les termes de la licence Creative Commons Attribution - Pas d'Utilisation Commerciale - Pas de Modification 4.0 International 


\title{
L'expérience du courriel en situation professionnelle : représentations de l'activité, jugements et affects
}

\author{
Nadia Gauducheau \\ Tech-CICO/ICD, Université de technologie de Troyes \\ 12, rue Marie Curie, BP 2060, 10010 Troyes Cedex \\ nadia.gauducheau@utt.fr
}

\begin{abstract}
The experience of email at work: Representations, judgments and affects. The objective of the paper is to describe the experience of email usage at work. Twenty five interviews inspired by "explicitation" and "self-confrontation" techniques were carried out with employees of a professional training company. These interviews allowed us to identify three dimensions of user experience: the representations of email activity, judgments on email (advantages, disadvantages) and the affects elicited by email usage. The analysis shows that the negative experience of users is linked to three phenomena. Firstly, the use of email is often associated with making it more difficult to control work activity. Secondly, email exchanges are seen as violating social and communicative rules. Finally, a lack of self-efficacy is sometimes experienced by users.
\end{abstract}

KEYWORDS

user experience, professional activity, electronic mail, affects, representations

\section{1.- Introduction et problématique}

L'analyse de l'expérience des utilisateurs est depuis une dizaine d'années considérée comme une approche fructueuse pour évaluer les nouvelles technologies (Light, 2006). Elle a donné lieu à un domaine d'étude (UX: user experience) comprenant différentes disciplines comme la psychologie, l'ergonomie (des logiciels), la psychologie ergonomique, le marketing ou l'informatique. Dans ce domaine, la compréhension du ressenti des utilisateurs paraît centrale pour comprendre l'acceptabilité des technologies (Hassenzahl, \& Tractinsky, 2006).

Nous proposons dans cet article d'étudier l'expérience des utilisateurs du courriel en situation professionnelle. ${ }^{1}$ L'usage du courriel nous semble être une situation intéressante pour deux raisons. Tout d'abord, peu d'études dans le domaine de l'expérience utilisateur portent sur des technologies familières (Kujula, Roto, Väänänen-Vainio-Mattila, Karapanos, \& Sinnelä, 2011). Ce sont plutôt les réactions des utilisateurs face à de nouveaux dispositifs qui font l'objet d'investigations (par exemple, de nouveaux services sur téléphone portable). Comme l'expérience face à un dispositif utilisé pour la première fois ou depuis peu n'est pas

Cet article s'inscrit dans un programme de recherche soutenu par l'ANR intitulé « Communication et multiactivité au travail (COMUT)» (ANR-08-COMM-039). 
la même que celle qui prend place lors d'un usage prolongé (Karapanos, Zimmerman, Forlizzi, \& Martens, 2009), il semble important d'étudier également des dispositifs connus des usagers et utilisés depuis une longue période. Ce type d'étude peut contribuer à l'identification des facteurs qui peuvent améliorer ou au contraire détériorer l'expérience des usagers sur le long terme. De plus, il existe un enjeu particulier à comprendre l'expérience des professionnels concernant le courriel puisque ses effets sur le travail et sur les salariés sont problématiques. Le courriel constitue un outil très utilisé dans les entreprises pour communiquer et collaborer. Cependant, il est à l'origine de problèmes de stress et de surcharge au travail (Mano, \& Mesch, 2010). Qu'en disent les usagers ? Quels sentiments leur inspire l'outil banal qu'ils utilisent quotidiennement, mais qui est apparemment source de problèmes dans leur travail ?

L'objectif de cette recherche est donc de décrire l'expérience du courriel par des professionnels (jugements, affects, sentiments...) et d'identifier les facteurs qui conduisent à une expérience de valence plutôt positive ou au contraire de valence négative. Elle a été réalisée auprès de salariés d'une entreprise de formation professionnelle qui conçoit et réalise des formations pour des entreprises dans divers domaines. Cette entreprise nous semblait intéressante, car le courrier électronique est installé et utilisé par tout le personnel depuis une dizaine d'années. Cela nous permettait donc d'avoir accès à des usagers habitués du courriel. De plus, la taille assez importante de l'entreprise permettait de rencontrer des salariés pour qui les enjeux et les effets du courriel peuvent être variés. Ainsi, nous avons interrogé des managers, souvent désignés comme les plus touchés par les méfaits du courriel, mais aussi des personnels avec des métiers pour lesquels les effets du courriel sont peu connus (service financier, $\mathrm{RH}$, etc.).

Nous présenterons, dans la première partie de cet article, la notion d'expérience utilisateur pour montrer qu'elle amène à s'intéresser aux représentations et aux affects des utilisateurs. Nous nous interrogerons ensuite sur le rôle des affects dans l'acceptation des technologies et sur les effets du courriel en situation de travail. Nous présenterons ensuite les entretiens et leur analyse. Nous décrirons ainsi les représentations de l'activité de traitement des messages par les professionnels, leurs jugements sur l'outil et les affects suscités par l'usage du courriel. Les résultats permettront de discuter des facteurs en jeu dans l'expérience des usagers du courriel.

\section{2.- Apports théoriques : expérience utilisateur et usage du courrier électronique au travail}

Cette partie présente la notion d'expérience utilisateur ainsi qu'une synthèse des travaux sur le rôle des affects dans l'acceptation des technologies et sur le courriel en entreprise.

\section{1.- L'expérience utilisateur : focalisation sur les affects et les représentations}

Les travaux sur l'expérience utilisateur (ou UX, user experience) présentent des objectifs multiples : la conception, l'évaluation de technologies ou de services et la compréhension de la relation homme-technologie. Un certain nombre de présupposés sont sous-jacents à une approche de type expérience utilisateur selon Hassenzahl et Tractinsky (2006). Nous avons choisi d'inscrire cette étude dans ce domaine, car ces principes nous semblent pertinents pour appréhender la relation homme-technologie et notamment la relation des individus au courrier électronique.

Tout d'abord, il s'agit de mettre en avant les limites d'une vision instrumentale de la relation homme-technologie (par exemple, ne pas se contenter d'étudier l'utilisabilité ou l'utilité d'une technologie, cf. Barcenilla, \& Bastien, 2009). De plus, il s'agit d'accorder une place importante aux affects et à la subjectivité dans les interactions homme-technologie (nous 
développerons le rôle des affects dans la partie suivante). Enfin, la notion d'expérience implique une conception de la relation homme-technologie comme située (elle se construit dans un contexte donné) et dynamique (elle évolue dans le temps). Selon Hassenzahl et Tractinsky (2006, p. 95), l'expérience est « une conséquence de l'état interne de l'utilisateur (prédispositions, attentes, besoins, motivation, humeur, etc.), des caractéristiques du système conçu (par exemple, complexité, but, utilisabilité, fonctionnalité, etc.) et du contexte (ou de l'environnement) dans lequel l'interaction se produit (par exemple, contexte social/organisationnel, signification de l'activité, caractère volontaire de l'utilisation, etc.) ». L'enquête de Law, Roto, Hassenzahl, Vermeeren et Kort (2009) confirme que les auteurs, dans le domaine de l'UX, s'accordent sur le caractère complexe, dynamique, subjectif de l'expérience utilisateur.

Malgré ce consensus, différentes approches apparaissent. Elles ne s'opposent pas nécessairement, mais se distinguent du point de vue des cadres théoriques mobilisés et des questions abordées. Nous proposons de présenter les deux qui nous semblent les plus souvent citées dans les travaux de psycho-ergonomie.

La première approche est celle proposée par McCarthy et Wright (2004) qui s'inspirent de la philosophie pragmatiste. Le travail de ces auteurs permet d'articuler la notion d'expérience en général avec celle d'expérience utilisateur. Pour ces auteurs, les technologies sont impliquées dans notre expérience de la vie quotidienne à la maison, au travail ("technology is deeply embedded in our ordinary everyday experience", p. 2). L'étude de la relation homme-technologie suppose donc de comprendre l'expérience des individus. Celle-ci est décrite comme un processus de construction de sens (sense making) continu et dynamique, qui s'appuie sur les attentes, les anticipations, l'histoire personnelle des individus et les interactions avec les objets. Les auteurs prônent une conception holistique du produit de l'expérience : elle est constituée de pensées, de sensations, et d'émotions qui forment un tout (flux continu). Une des limites de cette approche est qu'elle propose peu d'éléments pour étudier l'expérience utilisateur sur le plan opérationnel. Il s'agit plutôt d'un «manifeste » pour prendre en compte cet aspect dans la conception (Light, 2006).

D'autres auteurs proposent également de décrire le processus d'élaboration de l'expérience, mais avec un arrière-plan théorique différent. Ils s'inscrivent plutôt dans l'épistémologie de la psychologie expérimentale et analysent l'expérience utilisateur comme un processus de jugement ou d'évaluation qui prend place pendant ou après une interaction avec une technologie/un produit. Leur objectif est de déterminer les dimensions qui composent l'expérience utilisateur à un moment donné et leurs relations de codétermination. Au sein de cette seconde approche, plusieurs modèles, assez proches, ont été proposés.

Pour Mahlke et Thüring (2007) ${ }^{2}$, l'UX comporte trois composantes : la perception des qualités instrumentales des technologies (par exemple, contrôlabilité, efficacité, etc.), la perception des qualités non instrumentales (les qualités esthétiques, motivationnelles et symboliques de la technologie, par exemple, les valeurs associées) et les réactions émotionnelles (sentiments, réactions physiologiques, etc.). L'ensemble de ces trois composantes conduit à produire une évaluation du produit (avec des conséquences pour l'usage). Les propriétés du système, les caractéristiques de l'utilisateur et la nature de la tâche, résumées sous le terme de caractéristiques de l'interaction influencent également l'expérience de l'utilisateur.

Dans le modèle de Hassenzahl (2004, 2008), les individus perçoivent les attributs d'un produit en fonction de ses caractéristiques (contenu, fonctionnalités, présentation) et des attentes et normes personnelles. Deux catégories d'attributs sont distinguées : les qualités pragmatiques (liées à la tâche à réaliser comme l'utilisabilité) et les qualités hédoniques

2 Le modèle de Mahlke et Thüring (2007) intègre la plupart des dimensions de l'expérience que l'on trouve dans ce type d'approche selon Barcenilla, \& Bastien (2009). 
(liées au bien-être, au développement personnel de l'individu, par exemple permettre de satisfaire un besoin de nouveauté, associer le produit à une image sociale valorisante). Ces perceptions des qualités du produit ont comme conséquences que le produit sera plus ou moins attrayant/évalué positivement et suscitera des émotions (plus ou moins de plaisir et de satisfaction). La situation d'usage va influencer les perceptions ainsi que ses conséquences.

Enfin, pour Desmet et Hekkert (2007), l'UX comporte trois composantes: le plaisir esthétique (propriétés sensorielles des objets comme l'apparence ou le toucher), les significations attribuées à l'objet (propriétés symboliques, attentes associées à l'objet) et les émotions et sentiments suscités par l'objet. Ces composantes de l'expérience ont des mécanismes différents, mais peuvent s'influencer. De plus, elles sont déterminées par les caractéristiques de l'utilisateur (personnalité, compétences, buts et motivations, normes culturelles) et celles du produit/système.

Les modèles proposés dans cette seconde approche incluent comme déterminants de l'expérience utilisateur la situation, la culture ou la valeur symbolique de l'objet. Ils intègrent donc le caractère situé et complexe de l'UX. Néanmoins, ces aspects sont négligés dans les recherches empiriques qui sont, pour l'essentiel, centrées sur les relations entre les différentes composantes (entre jugement d'utilisabilité et jugement esthétique ou émotions) et qui portent sur des situations expérimentales simplifiées (un épisode isolé d'interaction avec une technologie nouvelle).

En nous appuyant sur l'ensemble de ces travaux, il nous semble possible de distinguer deux composantes centrales de l'UX : d'une part les représentations associées à la technologie et d'autre part les affects suscités par la technologie. Nous définissons les représentations sociales comme les jugements, attitudes et informations élaborés par les sujets à propos d'un objet (Abric, 1994). Le terme de représentation nous semble pertinent, car il permet d'intégrer deux aspects qui sont évoqués dans les modèles précédents, d'une part l'activité de construction de connaissance sur l'objet et son usage (construction d'un savoir «naiff » sur les propriétés de l'objet) et d'autre part l'activité d'évaluation (des jugements sur l'objet et la situation d'usage). De plus, utiliser la notion de représentation sociale permet de souligner le rôle de la relation à l'autre et du contexte social dans la manière de se représenter l'objet et la situation d'usage.

Les affects englobent les émotions, les humeurs et les sentiments (Cosnier, 1994). L'expérience affective (ce que la personne ressent ou éprouve) la plus élémentaire correspond à un sentiment d'attrait ou de rejet, de plaisir/déplaisir envers une situation, un objet (Feldman-Barrett, Mesquita, Ochsner, \& Gross, 2007).

On peut noter que le rôle des représentations associées aux nouvelles technologies avait déjà été souligné en sociologie des usages. Ces travaux ont montré que les représentations et les valeurs associées aux technologies permettent de comprendre la manière dont celles-ci sont intégrées dans les pratiques (Mallein, \& Toussaint, 1994).

Quant aux affects, un certain nombre de travaux en psychologie se sont attachés à montrer leur influence sur les interactions homme-machine et l'acceptabilité des technologies (voir Février, Gauducheau, Jamet, Rouxel, \& Salembier, 2011). De plus, par définition, l'émotion d'un individu reflète de manière privilégiée son évaluation d'une situation. En effet, la réaction émotionnelle est le résultat d'un processus d'appraisal (analyse, évaluation) de la situation et d'attribution de signification aux évènements notamment en fonction des buts et des intérêts de l'individu (Fridja, 1986; Scherer, 2001). Nous allons présenter plus précisément dans la partie suivante le rôle des affects dans l'acceptation des nouvelles technologies et produits.

\section{2.- Affects et acceptation des technologies}

Selon Norman (2004) et Jordan (2000), le rôle des affects est à prendre en compte au 
moment de la conception. Ces auteurs défendent le point de vue selon lequel l'usage des technologies permet de satisfaire des besoins affectifs : éprouver du plaisir, de l'amusement, de la stimulation (voir Barcenilla, \& Bastien, 2009 pour une présentation de ces travaux). L'objectif serait de concevoir des technologies (et produits) permettant de produire des émotions positives chez l'utilisateur et d'éviter les états émotionnels négatifs nuisibles à l'activité. Jordan (2000) propose par exemple une liste de plaisirs à susciter: des plaisirs physiologiques (liés aux organes sensoriels : toucher, esthétique agréable d'un produit...), des plaisirs idéologiques (répondre aux goûts, valeurs et aspirations de l'utilisateur), etc.

D'autres auteurs s'intéressent au rôle des affects des utilisateurs lorsqu'ils sont face à un nouveau produit, une nouvelle technologie. Selon Bagozzi et Lee (1999), l'évaluation d'une innovation peut produire des émotions positives (joie, fierté, espoir...) si celle-ci est jugée favorable à l'atteinte de buts personnels ou provoquer, au contraire, des émotions négatives (frustration, colère, peur...) si elle constitue une menace pour l'atteinte des buts de l'individu. Ces émotions ont alors un rôle dans l'adoption de l'innovation ou la résistance par les individus. La réaction affective des utilisateurs face à un nouveau produit peut avoir des conséquences sur leur attitude plus générale concernant la marque (Chaudhuri, \& Holbrook, 2001). Par exemple, les utilisateurs éprouvant une expérience affective positive ont plus souvent l'intention de racheter un produit de la même marque (Chitturi, Raghunathan, \& Mahajan, 2008 ; Garrett, 2006).

Finalement, de nombreux auteurs en arrivent à la même conclusion : il est préférable que l'utilisation d'une technologie ou d'un produit suscite des émotions positives plutôt que négatives. Cette hypothèse, qui semble aller de soi, est néanmoins limitée de notre point de vue. En effet, considérer que les émotions positives sont bénéfiques dans toutes les situations (et inversement pour les émotions négatives) pose un problème. Les relations entre cognition et émotion sont plus complexes. Par exemple, les affects positifs conduisent à prendre des décisions plus risquées, à utiliser des heuristiques plutôt qu'un raisonnement systématique (Isen, 2000). À l'inverse, des travaux montrent le rôle positif d'émotions négatives peu intenses, par exemple sur la capacité à produire des messages persuasifs pertinents ou à procéder par raisonnement systématique (Forgas, 2007). Wood et Moreau (2006) montrent également que l'impact des affects est complexe. Lorsque les individus rencontrent des difficultés inattendues pour apprendre à se servir d'un nouveau produit, cela provoque des émotions négatives (frustration, colère) qui ont un impact négatif sur l'évaluation globale du produit. Si ces difficultés sont attendues, les émotions négatives qu'elles suscitent n'ont pas d'impact sur l'évaluation. De plus, ces auteurs montrent que les émotions provoquées par la facilité/difficulté d'apprentissage du produit sont indépendantes de celles qui résultent de l'évaluation des bénéfices liés au produit. Elles peuvent coexister et être contradictoires. Enfin, une approche des affects en termes de valence (affect positif versus négatif) semble insuffisante pour comprendre leur rôle dans la cognition. Sur le plan théorique, cette distinction ne permet pas de rendre compte des émotions si l'on se réfère aux théories de l'appraisal (Scherer, 2001). En effet, deux émotions de même valence peuvent correspondre à des évaluations de la situation (appraisals) différentes. Par exemple, dans le cas de la peur, la situation est perçue comme incontrôlable, les événements négatifs sont perçus comme imprévisibles et liés aux contraintes de la situation. Dans le cas de la colère, la valence de l'émotion est négative comme pour la peur, mais l'évaluation de la situation est différente. L'individu la perçoit comme contrôlable et les évènements négatifs comme prévisibles (Lerner, \& Keltner, 2000). Lerner et Keltner (2000) montrent que ces différences ont un impact sur la perception des risques. Les individus éprouvant de la peur font des prévisions pessimistes alors que les individus éprouvant de la colère font des prévisions optimistes.

Comme nous l'avons évoqué dans l'introduction, l'expérience des usagers du courriel n'a pas fait l'objet d'études spécifiques puisque les travaux sur l'UX ont privilégié l'analyse de dispositifs nouveaux. En revanche, de nombreux résultats sur l'usage du courriel en situation de travail sont disponibles. 


\section{3.- Le courriel au travail}

L'introduction du courriel en entreprise a eu un impact sur l'activité des professionnels, notamment sur la gestion des tâches, l'efficacité et l'émergence de contraintes.

Le plus souvent, les études pointent les effets néfastes de l'usage du courriel. Un des problèmes est la quantité trop importante de messages reçus à traiter. Cela provoque chez certains usagers un sentiment de surcharge informationnelle et communicationnelle (Whittaker, \& Sidner, 1997).

Le courriel contribue, de manière générale, à une intensification du travail. Tout d'abord, l'arrivée incessante de nouveaux messages constitue une source d'interruptions et de fragmentation de l'activité (Assadi, \& Denis, 2005). Jackson, Dawson et Wilson (2001) montrent que l'arrivée d'un courriel interrompt la tâche en cours, car celui-ci suscite le plus souvent une réponse rapide (souvent dans les $5 \mathrm{mn}$ ). Dans l'étude de Czerwinski, Horvitz, et Wilhite (2004), les utilisateurs rapportent traiter leur courriel, de manière répétée, tout au long de la journée. Cette tâche représente $23 \%$ de leur temps de travail.

Le courriel instaure également une pression temporelle: un sentiment d'urgence, une augmentation de la « joignabilitée ${ }^{3} »$ des salariés (Besseyre des Horts, 2008) et une incitation à être encore plus réactif durant le travail voire au domicile (Assadi, \& Denis, 2005). Ainsi, l'usage du courriel se rapproche, en entreprise, de celui d'une messagerie synchrone. Cette pression temporelle peut être source de stress (Hair, Renaud, \& Ramsay, 2007).

Enfin, l'introduction de la messagerie engendre également de nouvelles tâches de manutention de l'information (Issac, Campoy, \& Kalika, 2007) comme le tri et le classement des messages (Ducheneaut, \& Watts, 2005) qui s'ajoutent aux activités habituelles.

Ces tâches impliquent de développer des savoir-faire spécifiques liés au traitement des messages (faire des dossiers, savoir hiérarchiser de manière efficace). Elles peuvent être perçues comme une surcharge de travail ou une forme de déqualification du travail lorsqu'elles sont considérées comme en dehors du cœur de métier (Datchary, \& Licoppe, 2007). Les salariés, en gérant un travail fragmenté par les interruptions, peuvent également être amenés à développer un savoir-faire de «dispersion» (Datchary, 2009). Russell, Purvis, et Banks (2007) observent que les individus développent des stratégies pour contrôler ces interruptions. Selon l'importance du message et la tâche en cours, le message sera ou non traité, il y aura ou non un retrait de l'attention engagée dans l'activité en cours.

Par ailleurs, quelques travaux mettent en avant des problèmes de communication comme l'incertitude sur les conventions d'usage, par exemple sur le délai de réponse attendu (Assadi \& Denis, 2005), ou encore le caractère agressif de certains messages lié notamment à l'absence de formalisme (Akrich, Meadel, \& Paravel, 2000).

Certaines études, focalisées sur l'utilité du courriel, montrent, au contraire, les avantages de l'usage du courriel. Il permet notamment d'améliorer la transmission des informations entre professionnels, la collaboration à distance, l'archivage des informations (Ducheneaut, \& Bellotti, 2001). Mano et Mesch (2010) observent un lien entre l'intensité de l'usage du courriel et la performance des salariés: plus les individus consultent souvent leur boîte, envoient et reçoivent des messages, plus ils sont performants. O'Kane et Hargie (2007) montrent que la perception des employés est en partie cohérente avec ces travaux: les usagers considèrent que le courriel présente certains avantages par rapport au face à face (par exemple, être plus facilement informé sur l'entreprise).

3 La joignabilité « représente la capacité pour un individu d'être joint et de joindre les autres facilement, et ceci avec un minimum de contraintes » (Besseyre des Horts, 2008, p. 134). 
Ces études mettent en avant, pour l'essentiel, les problèmes du courriel pour la gestion de l'activité professionnelle et plus rarement les avantages de l'outil. Elles ne permettent pas réellement de rendre compte de la perception et des ressentis des usagers, mais suggèrent que leur l'expérience serait plutôt négative (notamment, à cause du sentiment de surcharge). Nous proposons dans cette étude d'approfondir cette question en analysant spécifiquement l'expérience des usagers, c'est-à-dire en étudiant leurs représentations et leurs affects.

\section{3.- Méthodologie}

\section{1.- Terrain}

Cette étude a été réalisée dans une grande entreprise de formation professionnelle (formation de type e-learning, séminaires, conseil...) qui comporte environ 1200 salariés. Les formations sont assez variées : bureautique, marketing, management, ressources humaines, formations liées à des secteurs spécialisés (automobile, banque...).

Vingt-cinq entretiens auprès de différents types de personnels ont été réalisés sur leur lieu de travail pendant environ une heure (13 femmes et 12 hommes). L'échantillon a été constitué en vue de représenter différents métiers (la formation, mais aussi, le service qualité, le marketing, la logistique, la comptabilité) et différents niveaux hiérarchiques (par exemple, manager/assistante) (voir Tableau 1). Les participants sont employés dans l'entreprise depuis 10 ans en moyenne (voir Tableau 2).

Le courrier électronique a été installé dans cette entreprise il y a une quinzaine d'années auprès des managers puis s'est généralisé rapidement à tout le personnel. Les participants ont estimé le nombre de messages reçus et envoyés par jour. Des écarts importants sont apparus : ils estiment recevoir de 10 à 70 messages et envoyer de 10 à 50 messages par jour (voir Tableau 2). Le nombre de messages semble lié à la position hiérarchique. Les managers reçoivent généralement beaucoup de messages (cf. Mano, \& Mesch, 2010 ; dans cette étude ils en reçoivent en moyenne 43 par jour). La nature de l'activité professionnelle peut aussi influencer le nombre de messages. Par exemple, les consultants-formateurs échangent par courriel avec de nombreux interlocuteurs, en interne pour organiser les formations et en externe pour gérer les relations commerciales.

\begin{tabular}{|c|c|c|}
\hline Type de service & $\begin{array}{c}\text { Niveau hiérarchique faible } \\
\text { ou moyen (16) }\end{array}$ & $\begin{array}{c}\text { Niveau hiérarchique plus } \\
\text { élevé (responsabilité, } \\
\text { encadrement d'une équipe) } \\
(9)\end{array}$ \\
\hline $\begin{array}{lll}\text { Relation } & \text { commerciale } & \text { et } \\
\text { marketing } & & \end{array}$ & $\begin{array}{l}\text { Commercial (1) } \\
\text { Chargé d'étude marketing (1) }\end{array}$ & Managers (2) \\
\hline $\begin{array}{l}\text { Unités par offre de formation } \\
\text { (unité management, unité } \\
\text { commerce international...) }\end{array}$ & $\begin{array}{l}\text { Assistant de direction (2) } \\
\text { Assistant (1) } \\
\text { Consultant-formateur (5) } \\
\text { Conceptrice multimédia (1) }\end{array}$ & Managers (7) \\
\hline Logistique & $\begin{array}{l}\text { Gestionnaire (salles, } \\
\text { organisation des formations) } \\
\text { (1) }\end{array}$ & \\
\hline Qualité & Responsable qualité (1) & \\
\hline Ressources Humaines & $\begin{array}{l}\text { Assistant de direction (1) } \\
\text { Responsable recrutement (1) }\end{array}$ & \\
\hline Comptabilité & Responsable comptabilité (1) & \\
\hline
\end{tabular}

Tableau 1 : Type de poste occupé par les participants (personnel sédentaire signalé en italique et effectif indiqué entre parenthèses)

Table 1: Types of job held by participants 


\begin{tabular}{|l|c|c|c|c|}
\hline & Moyenne & Écart type & Min. & Max. \\
\hline $\begin{array}{l}\text { Ancienneté dans } \\
\text { l'entreprise }\end{array}$ & 10,5 & 5.4 & 4 & 18 \\
\hline $\begin{array}{l}\text { Nbre de messages } \\
\text { reçus/jour }\end{array}$ & 32 & 16.5 & 10 & 70 \\
\hline $\begin{array}{l}\text { vbre de messages } \\
\text { envoyés/jour }\end{array}$ & 26 & 15 & 10 & 50 \\
\hline
\end{tabular}

Tableau 2 : Caractéristiques des participants

Table 2: Participants' characteristics

\section{2.- Entretiens}

Nous avons choisi de réaliser des entretiens pour étudier l'expérience des usagers, dans la lignée des travaux de Cahour, Brassac, Vermersch, Bouraoui, Pachoud et Salembier (2007) ou Light (2006). L'entretien semble être une méthode pertinente pour étudier l'expérience puisqu'il s'agit d'avoir accès au ressenti des utilisateurs. Comme l'affirment FeldmanBarrett et al. (2007), il semble difficile de ne pas faire appel à un processus réflexif et langagier lorsque l'on souhaite avoir accès à l'expérience émotionnelle.

Ces entretiens s'inspirent des techniques d'explicitation et d'auto-confrontation qui visent à la verbalisation de l'activité vécue en incitant les participants à se remémorer leur activité dans leur contexte. L'entretien d'explicitation cherche à guider le sujet dans la verbalisation précise de sa conduite, des actions matérielles ou mentales réalisées (Vermersch, 1991). Il consiste à aider le sujet à se mettre en évocation de la situation qu'il a vécue en l'amenant progressivement à en retrouver le contexte sensoriel (ce qu'il voyait, entendait...) et environnemental (à quel endroit...) en le questionnant sans induire un contenu spécifique. L'entretien d'auto-confrontation simple consiste à confronter un participant à sa propre activité en l'incitant à la commenter (Mollo, \& Falzon, 2004).

L'objectif des entretiens réalisés dans cette étude était d'obtenir des descriptions détaillées de l'activité vécue. En référence à Theureau (2000), nous considérons «l'activité de travail comme constituant un tout dynamique (ne séparant pas, comme c'est souvent fait, émotion, attention, perception, action, communication et interprétation)» (p. 182). En nous focalisant sur l'activité, nous visions donc par la même occasion les ressentis et perceptions des usagers.

Concrètement, quelques questions générales ont été posées afin de connaitre le profil des participants (poste et missions, terminaux et logiciels utilisés pour lire les messages, etc.). Ensuite, dans la première phase de l'entretien, inspirée des techniques d'explicitation, les participants ont été invités à décrire le plus précisément possible leur usage du courriel. Deux thèmes ont été abordés: le déroulement de l'activité de traitement des messages (consultation, tri, sélection, réponse, archivage) et la rédaction des messages (stratégies). L'objectif était d'aborder l'usage du courriel selon deux postures : celle de récepteur et celle d'émetteur. Les relances visaient à inciter les participants à détailler leur activité et à donner des exemples de situations. Nous avons laissé le sujet décrire d'abord ses procédures en général puis nous lui demandions de décrire des situations précises. Des exemples de questions utilisées (consignes, relances) sont présentées dans le tableau 3. 


\begin{tabular}{|c|c|c|}
\hline Thème & $\begin{array}{c}\text { Exemple de question de } \\
\text { départ (consigne) }\end{array}$ & Exemple de relance \\
\hline \multicolumn{3}{|c|}{ Première phase de l'entretien (inspiré de l'explicitation) } \\
\hline $\begin{array}{l}\text { Déroulement de } \\
\text { l'activité de } \\
\text { traitement des } \\
\text { messages }\end{array}$ & $\begin{array}{l}\text { - Comment vous gérez } \\
\text { l'activité de lecture, traitement } \\
\text { des mails dans votre journée de } \\
\text { travail? } \\
\text { - Pourriez-vous expliquer } \\
\text { comment vous procédez pour } \\
\text { traiter vos messages? }\end{array}$ & $\begin{array}{l}\text { - Et une fois que vous ouvrez le mail } \\
\text { y a-t-il d'autres éléments qui } \\
\text { peuvent attirer votre attention? } \\
\text { - Et comment vous faites gaffe? } \\
\text { (suite aux propos suivants .... si on } \\
\text { ne fait pas gaffe, on peut se faire } \\
\text { déborder...) } \\
\text { - Avez-vous un exemple? }\end{array}$ \\
\hline $\begin{array}{l}\text { Pratiques de } \\
\text { rédaction d'un } \\
\text { message }\end{array}$ & $\begin{array}{l}\text { - Pouvez-vous essayer de } \\
\text { préciser votre } \\
\text { démarche lorsque vous rédigez } \\
\text { un mail ? Pouvez-vous me } \\
\text { donner un exemple? }\end{array}$ & $\begin{array}{l}\text { - Et dans le corps du texte, y a-t-il } \\
\text { d'autres choses auxquelles vous êtes } \\
\text { attentif? } \\
\text { - Reformulation : vous lui avez } \\
\text { répondu très rapidement? (suite aux } \\
\text { propos suivants .... je lui ai répondu } \\
\text { dans la minute...). }\end{array}$ \\
\hline \multicolumn{3}{|c|}{ Seconde phase de l'entretien (inspiré de l'autoconfrontation) } \\
\hline $\begin{array}{l}\text { Commenter les } \\
\text { messages reçus }\end{array}$ & $\begin{array}{l}\text { - Vous pouvez lire vos } \\
\text { messages, m'expliquer ce que } \\
\text { c'est et ce que vous en avez } \\
\text { fait ou allez en faire? }\end{array}$ & $\begin{array}{l}\text { - Comment faites-vous pour savoir } \\
\text { que vous avez ce mail à traiter ou } \\
\text { bien que vous avez une action à } \\
\text { faire sur ce point-là? }\end{array}$ \\
\hline $\begin{array}{l}\text { Commenter les } \\
\text { messages envoyés }\end{array}$ & $\begin{array}{l}\text { - J'aimerais maintenant que } \\
\text { nous regardions les mails que } \\
\text { vous avez envoyés } \\
\text { - Pouvez-vous m'expliquer ce } \\
\text { que vous avez mis dans ce } \\
\text { mail? }\end{array}$ & $\begin{array}{l}\text { - Pouvez-vous m'expliquer à quoi } \\
\text { vous avez été attentif quand vous } \\
\text { avez écrit ce mail ? } \\
\text { - Et quand vous lui dites «j'ai peur } \\
\text { de me répéter », ça veut dire quoi? }\end{array}$ \\
\hline
\end{tabular}

Tableau 3 : Exemples de consignes et relances dans les entretiens

\section{Table 3: Examples of instructions and follow-up questions in interviews}

Dans une seconde phase de l'entretien, inspirée des techniques d'auto-confrontation, il était demandé aux participants de commenter les derniers messages reçus et envoyés (cela impliquait souvent de commenter l'ensemble de la discussion). Les derniers messages des boîtes de réception et d'envoi ont été commentés les uns après les autres par ordre chronologique. Nous avons invité les participants à expliciter les actions réalisées à la réception de ces messages, les raisonnements qui les ont conduits à répondre ou ne pas répondre au message, à les archiver, à la manière de rédiger leurs propres messages, etc. (voir Tableau 3). Nous avons choisi d'utiliser comme traces de l'activité les messages les plus récents afin de favoriser la remémoration de l'activité. Le nombre de messages commentés variait selon les participants en fonction du temps passé sur chacun (certains messages ont suscité plus de verbalisations que d'autres) et du temps disponible (nous nous étions engagée à ne pas mobiliser les participants plus d'une heure).

\section{3.- Analyse}

Une analyse de contenu thématique des entretiens a été réalisée afin de repérer les thèmes pertinents dans le discours des participants, en lien avec les objectifs de la recherche (Paillé, \& Muchielli, 2003). Il s'agissait de rendre compte de l'expérience des usagers c'est-à-dire de leurs représentations et de leurs affects. Trois dimensions de l'expérience sont apparues pertinentes. La première correspond aux représentations de l'activité de traitement des messages et renvoie aux descriptions des activités menées par les participants lorsqu'ils utilisent leur messagerie, et à leur contexte. La seconde dimension correspond aux propos évaluatifs des usagers concernant le courriel (avantages, désavantages). Cette dimension 
rejoint en partie ce qui est appelé jugements des utilisateurs sur les qualités pragmatiques de l'outil dans les travaux sur l'UX. La troisième dimension correspond aux affects suscités par l'usage du courriel et reprend les propos des participants décrivant leurs états affectifs ainsi que le contexte de leur apparition.

Plus précisément, pour les représentations de l'activité de traitement des messages, nous avons procédé à une analyse thématique classique en repérant les sous-thèmes récurrents et en opérant des distinctions/regroupements.

Pour les jugements sur l'outil, les thèmes ont été classés en deux catégories opposées : les jugements plutôt positifs (propos décrivant ce que l'outil permet de faire «bien» ou «facilement») et les jugements plutôt négatifs (propos sur les limites, les conséquences négatives et les risques associés à l'outil).

Enfin, pour les affects, les descriptions d'états émotionnels ont fait l'objet d'une analyse lexicale. Nous avons utilisé la grille de codage de Scherer (2005, Geneva Affect Label Coder) qui permet d'identifier 36 état affectifs. Par exemple, cette grille classe dans « irritation» les mots commençant par irrit $^{*}$, agac $^{*}$, contrari $^{*}$, embêt $^{*}$, ennuy*, etc. La grille a été complétée en y ajoutant les termes familiers ou argotiques non présents. Par exemple, le verbe « barber» n'apparait pas dans la grille, mais, considéré comme synonyme du verbe «ennuyer», il a été classé dans «irritation». De même, l'expression «pêter un câble » a été classée dans «irritation» considérant qu'elle signifie "s'énerver», "être agacé ».

Nous avons également identifié les circonstances d'apparition des affects ou, autrement dit, les antécédents (ou causes) associés à chaque état affectif décrit.

Nous avons comptabilisé, pour chaque catégorie d'analyse, le nombre de participants dont des propos ont été classés dans la catégorie.

\section{4.- Résultats}

\section{1.- Représentations de l'activité de traitement des messages}

Lorsqu'ils décrivent leur activité de traitement du courriel, les participants évoquent généralement trois thèmes : leur engagement dans cette activité, les fonctions du courriel (à quoi leur sert le courriel ?) et les compétences associées à l'usage du courriel. Le tableau 4 indique le nombre de participants dont les propos ont été classés dans chaque catégorie.

\begin{tabular}{|l|c|}
\hline \multicolumn{2}{|c|}{ Engagement dans l'activité } \\
\hline Engagement permanent & 19 \\
\hline Engagement par intermittence & 6 \\
\hline \multicolumn{2}{|c|}{ Fonctions du courriel } \\
\hline Archivage compétences associées & 20 \\
\hline Envoi de messages/documents & 5 \\
\hline Impliquer plusieurs participants & 6 \\
\hline Amorce pour un échange (en position de réception et/ou d'émetteur) & 4 \\
\hline \multicolumn{2}{|c|}{} \\
\hline Perception d'un manque de compétences & 9 \\
\hline Sentiment d'être compétent & 6 \\
\hline
\end{tabular}

Tableau 4 : Représentations de l'activité de traitement des messages

Table 4: Representation of email activity 


\subsection{1.- Engagement dans l'activité}

Deux types de profils sont observés concernant l'engagement dans l'activité de traitement des courriels : l'engagement permanent et l'engagement par intermittence.

Le premier profil, d'engagement permanent, correspond à la majorité des participants (19). Les individus se perçoivent comme engagés en permanence dans l'activité de traitement des messages. En effet, ils se rendent disponibles pour l'arrivée de nouveaux messages en laissant leur messagerie ouverte en permanence et en consultant les nouveaux messages dès qu'ils sont signalés. En revanche, ils ne répondent pas toujours dans l'immédiat (Je le regarde plutôt au fil de l'eau. Je ne les traite pas forcément au fil de l'eau. Je n'ai pas pour habitude de me déconnecter). Cet engagement continu se manifeste chez des professionnels de différents statuts et métiers, aussi bien sédentaires (par exemple, responsable RH, comptabilité) que mobiles (par exemple, consultant-formateur, managers). Pour les personnels mobiles, cela signifie que le courriel est consulté dans divers lieux et non pas uniquement dans leur entreprise, par exemple, sur le site où ils dispensent une formation, chez des clients (sur smartphone ou PC portable). L'engagement continu de ces personnels implique quelques aménagements dans les moments de mobilité : les messages sont traités pendant les pauses par exemple. Mais, il s'agit bien d'un engagement permanent dans la mesure où les individus cherchent à consulter leurs messages le plus fréquemment possible.

Parmi ces individus engagés en permanence, certains déclarent également être particulièrement réactifs aux messages comme si l'outil était perçu comme un moyen de communication synchrone (10) (J'en laisse quelques-uns en attente. Mais très généralement, on répond assez vite. Je réponds très vite en fait). Par ailleurs, une fois la boîte de réception ouverte, les propriétés de l'outil semblent jouer un rôle important pour ces utilisateurs dans l'attention portée aux nouveaux messages. En effet, la messagerie est perçue comme un attracteur cognitif (Assadi, \& Denis, 2005 ; Lahlou, 2000). Les indices sonores et les fenêtres signalant l'arrivée d'un message captent l'attention des personnes (8) (C'est facile, il y a juste à cliquer sur un bouton. (...) De toute façon dès que je reçois un mail, il s'affiche sur mon écran).

Le second profil, qui concerne le reste des participants (6), est un engagement par intermittence. Dans ce profil, les participants déclarent contrôler le temps accordé au traitement du courrier électronique, notamment en l'adaptant aux tâches en cours. La messagerie est mise de côté lorsqu'une activité nécessite un engagement intense (tâche urgente, difficile, importante, etc.). Les participants réalisent un traitement des messages en début et/ou en fin de journée. Ils semblent donc se préserver du caractère intrusif de la messagerie (En période d'activité très dense, je me donne, par exemple, jusqu'à 9 h 30 le matin pour traiter les mails. (...) Non, je ferme la boîte, comme cela plus rien ne peut me parvenir). Parmi six professionnels avec ce profil, quatre sont managers. Pour ceux-là, nous faisons l'hypothèse qu'ils manifestent à travers cette attitude leur maîtrise du dispositif de communication (contrôle). En effet, les résultats suivants (4.1.3) indiquent que la maitrise du courriel pourrait représenter, pour les managers, un des enjeux de leur métier.

\subsection{2.- Fonctions du courriel}

Quatre fonctions du courriel ont été évoquées par les participants : archiver, envoyer des messages et des documents, impliquer plusieurs participants et amorcer un échange. Le poste occupé ne semble pas jouer de rôle particulier dans les fonctions assignées au courriel.

Les participants partagent la même représentation du rôle central de la messagerie comme outil de réception, plus précisément comme outil d'archivage des communications et des documents (20) (Si c'est quelque chose qui concerne mon métier, je garde (...) et je peux, au besoin, ressortir un message en lui disant qu'elle l'avait reçu puisqu'elle m'avait remercié). De manière générale, la conservation et le classement des messages constituent un support à l'organisation du travail (par exemple, par la visualisation des dossiers en cours). Par 
ailleurs, les participants évoquent souvent l'importance de l'archivage pour garder des preuves du travail réalisé ou des communications (cf. O'Kane, \& Hargie, 2007) (X est celui qui chapote l'ensemble. Sur ce message il a validé mes objectifs. Je garde sa validation. J'ai gardé sa validation non pas parce qu'elle m'est utile pour l'action, mais par exemple quand j'ai sorti les objectifs il m'a dit: "attention, je n'ai pas validé cela». Dans ce cas je lui réponds que je lui ai envoyé un message et qu'il a bien validé l'ensemble et je peux ressortir le mail pour en apporter la preuve. A ces postes dans lesquels ces personnes sont, ils reçoivent énormément de mails et traitent beaucoup de choses dans la journée. Je sais qu'ils ne peuvent pas se souvenir de tout).

Le courriel est également utilisé de manière évidente pour envoyer des messages et des documents (5) (Pareil en interne, par exemple il y a des bons de commande pour réservation de matériel, ça se passe par mail, et le bon de commande passe par mail). Il permet d'impliquer plusieurs participants en même temps (message à plusieurs destinataires) notamment afin de donner de l'importance à un message (6) (Je mets copie à la $n+2$ (..) pour avoir un impact psychologique on va dire/Nous devons évaluer la qualité de notre prestation "Il faut mettre en place un système d'évaluation de ce module » [lit le message reçu]. J'ai rencontré le patron $X$ qui m'a dit que je devais travailler avec d'autres personnes et qu'il fallait avancer, car il souhaitait donner comme objectif d'atteindre $80 \%$ de satisfaction de la clientèle. J'ai donc envoyé ce message aux personnes concernées : "Suite $\grave{a}$ un entretien avec $X$, notre patron, et conformément à nos objectifs qualités je vous propose de travailler. Je vous remercie de faire part de vos disponibilités » [lit le message envoyé]. Ils m'ont tous répondu dans la journée ou le lendemain. Je n'ai pas conservé ces réponses, mais cela a été très rapide).

Le fait qu'un courriel permette d'attester d'une action réalisée («preuve ») ou de légitimer une demande (en impliquant certaines personnes) incite les usagers à multiplier et conserver les messages. Ainsi, certaines fonctions du courriel jugées utiles sont en même temps sources de surcharge. De même, le fait de mettre en copie un supérieur peut engendrer des tensions (cela suscite l'irritation, cf. partie 4.3.1).

Enfin, le courriel permet également d'amorcer un échange, c'est-à-dire de signaler à autrui le souhait d'échanger avec lui par d'autres moyens (4) (Je voudrais qu'il m'appelle. D'ailleurs je lui écris : " appelle-moi » et je lui propose qu'il vienne me voir).

\subsection{3.- Des compétences associées}

Quinze participants ont évoqué les compétences associées à l'usage du courriel. Deux types de compétences sont mises en avant, celles relatives à l'usage du courriel en position de réception (classer, traiter les messages reçus) et celles associées à la position d'émetteur ou de communicant (rédiger un message). Ces participants se sont, à chaque fois, positionnés sur leur maîtrise de ces savoir-faire (maîtrise versus non-maitrise).

Ainsi, certains considèrent qu'ils ne sont pas compétents (9) comme «récepteurs », pour la gestion de l'information (8). Ils évoquent une mauvaise connaissance des fonctionnalités et une gestion de la boîte non optimale (pas de maîtrise ou d'usage des fonctionnalités comme les filtres ou les indices de suivi des messages, etc.) (Mais j'ai beaucoup trop de trucs, je le sais. Il faudrait que je fasse un truc de suivi. Mais je suis très mauvaise, on nous a pas formés en fait). Certains ne se sentent pas compétents comme "communicants », dans la rédaction des messages, dans la maîtrise du format de communication (3 dont 2 évoquant aussi leurs lacunes comme "récepteurs ») (Nous continuons avec un mail qui fait suite à plusieurs autres. Voilà le premier que j'ai fait: "j'ai oublié de te demander... » [lit le message envoyé] J'ai écrit parce que j'avais essayé de l'appeler et je n'arrivais pas à le joindre. Il venait de quitter mon bureau, il devait être dans les couloirs. J'ai écrit comme je parle, il ne peut pas comprendre. Ce n'est pas efficace. Je ne crois pas du tout à ce genre de mail (...). Parce qu'il ne va pas avoir le temps, il faudrait qu'il suive ma pensée. Il vaudrait mieux que je lui reparle). 
A l'inverse, d'autres salariés (6) se perçoivent comme compétents dans l'usage du courriel en tant que «récepteurs» (3), notamment en mettant en évidence une maîtrise de leur « joignabilité » et de l'archivage des messages (Pour moi c'est un outil de communication et d'information. Qui dit outil, dit utilisation, donc maîtrise). Le sentiment de compétence porte également sur la rédaction de messages et sur les stratégies de communication (4 dont 1 personne évoquant aussi ces compétences en réception) (Donc je sais que c'est une bonne stratégie. Quand je leur mets les feuilles de temps, ils savent et je sais qu'ils vont cliquer).

Tous les managers de cette étude ont évoqué les compétences liées à l'usage du courriel. Cinq jugent qu'elles sont insuffisantes et quatre se sentent au contraire compétents. Ceux qui se considèrent compétents ont également déclaré un engagement par intermittence dans l'activité de traitement des courriels. Ces résultats suggèrent que pour les managers la maîtrise de l'usage du courriel est davantage un objet de préoccupation que pour les autres professionnels. Nous faisons donc l'hypothèse que la maîtrise des TIC est perçue comme un enjeu important pour leur métier.

Dans les entretiens, les individus évoquent aussi les tâches et stratégies mises en œuvre pour traiter leurs messages (hiérarchisation du traitement en fonction des tâches en cours, limitation du nombre de messages non traités dans la boîte de réception, etc.). Nous ne détaillerons pas ce point, car il s'agit d'un domaine bien étudié (Ducheneaut, \& Watts, 2005).

Cette partie montre que l'usage du courriel est une activité intense, car elle implique le plus souvent d'être en permanence attentif (aux messages entrants) et nécessite des compétences spécifiques parfois non maitrisées. Les descriptions de l'usage du courriel par les salariés peuvent être accompagnées de descriptions des ressentis, mais nous ne les avons pas traités dans cette phase. Ces ressentis sont analysés dans la partie suivante qui rend compte des propos évaluatifs des participants (considèrent-ils le courriel comme pratique, utile et/ou néfaste ?).

\section{2.- Jugements sur le courriel}

Nous avons regroupé les jugements positifs dans la même catégorie. Elle correspond aux propos décrivant les avantages du courriel: ce qu'il permet de faire «bien» ou «facilement». Trois catégories de jugements négatifs ou désavantages du courriel ont été distinguées : le sentiment de charge qui correspond à une appréciation négative de la quantité de messages (c'est trop!), les risques associés à l'usage du courriel ainsi que ses limites (voir Tableau 5 : effectifs associés à chaque catégorie).

\begin{tabular}{|c|c|}
\hline \multicolumn{2}{|c|}{ Jugements positifs } \\
\hline Rapidité des échanges & 4 \\
\hline Diffusion de l'information & 3 \\
\hline Libre organisation de son travail & 2 \\
\hline Archivage & 2 \\
\hline Temps pour la rédaction & 1 \\
\hline Mobilité & 1 \\
\hline \multicolumn{2}{|c|}{ Jugements négatifs } \\
\hline Sentiment de charge & 13 \\
\hline Risque de conflit et/ou de mauvaise compréhension & 14 \\
\hline Risque de débordement & 9 \\
\hline Diffusion de messages non pertinents & 7 \\
\hline Limites pour la communication de proximité & 7 \\
\hline Limites pour le traitement des urgences & 7 \\
\hline
\end{tabular}

Tableau 5 : Jugements sur le courriel

Table 5: Judgments on email 


\subsection{1.- Les jugements positifs}

Un certain nombre d'avantages dans l'utilisation du courriel a été évoqué par les participants : rapidité des échanges, diffusion de l'information, libre organisation de son travail, archivage, temps de rédaction, mobilité.

Le courriel favorise, selon certains participants, la rapidité des échanges (4) (Comme la communication est simplifiée parce que les formules ne sont pas lourdes, on peut vite réagir (...). Pour moi, le mail est plutôt bénéfique dans la communication).

Il facilite également la diffusion des informations, par exemple grâce à la fonction copie qui permet d'informer les personnes moins concernées par un message (3) (C'est bien de mettre en copie tous les gens qui sont concernés d'assez près par le sujet traité même s'ils n'ont rien de spécial à faire).

Le caractère asynchrone des échanges permet la libre organisation de son travail notamment, car le courriel permet de consulter/traiter les messages au moment choisi (2) (Je préfère le mail, je trouve cela moins intrusif, la personne le lit quand elle le veut, si elle le veut, il y a une trace. Je trouve cela plus simple).

La possibilité de stockage et d'archivage des messages est parfois appréciée (2) (Je trouve cela plus simple. C'est important de tracer quand un animateur nous donne son accord pour une animation (...) Je trouve le mail super pratique).

Pour une personne, le caractère éditable des messages facilite la réflexion et la rédaction $(O n$ prend le temps de l'écriture, de la rédaction, de reformuler, on peut effacer, il y a tout un travail de création alors qu'une fois que c'est lâché oralement, c'est fini).

Enfin, pour une autre, la possibilité d'un usage du courriel en mobilité est un point positif (le fait de pouvoir lire ces mails sur le téléphone c'est pratique).

On remarque que les jugements positifs sont hétérogènes et très peu consensuels. La rapidité des échanges, évoquée par quatre personnes, est le jugement positif le plus partagé.

\subsection{2.- Sentiment de charge}

La moitié des participants juge que le flux des messages est important (13) (Je crois que c'est noyé après dans la masse du nombre de mails reçus parce qu'on en reçoit énormément par jour). Ce jugement apparaît chez différentes catégories de personnels. La perception d'une quantité importante de messages n'est pas nécessairement en relation avec le nombre de messages reçus. On trouve ce type de propos chez des personnes déclarant un nombre de messages plus ou moins important (des personnes déclarant recevoir 20 à 25 messages reçus par jour comme des personnes déclarant en recevoir 50). Cette observation témoigne du caractère subjectif de la perception de la surcharge de messages qui ne peut pas se réduire à une question de quantité ou de flux (Assadi, \& Denis, 2005).

\subsection{3.- Risques liés à l'usage du courriel}

Trois risques liés à l'usage du courriel sont évoqués : le risque de conflit et/ou de mauvaise compréhension, le risque de débordement et celui de diffusion de messages non pertinents, inutiles.

Le problème le plus souvent associé à l'usage du courriel est le risque de conflit et/ou de mauvaise compréhension des messages (14) (Je fais attention parce que le mail (...) c'est quelque chose qui peut vite partir dans l'échange, les gens peuvent vite s'agacer pour des mots qu'ils auraient interprétés et qui ne sont pas forcément dans mon intention). Ce risque fait écho aux limites que l'on prête à la communication médiatisée par ordinateur (CMO) dans la littérature. Notamment, Sproull et Kiesler (1986) proposent l'hypothèse selon laquelle l'accès limité au contexte (activités en cours, contexte de travail, etc.) ou encore l'absence d'indices non verbaux (mimiques, intonation, etc.) seraient à l'origine de conflits ou de malentendus dans la CMO. Autrement dit, il serait plus difficile de faire passer des 
messages complexes, sophistiqués dans ces conditions. Ce risque est évoqué par presque tous les managers (8 sur les 9 ). Plusieurs hypothèses peuvent expliquer ce phénomène. La première est que leur expérience du courriel les a amenés à faire ce constat. Leur profession implique sans doute souvent de négocier et de gérer des conflits par courriel. La seconde hypothèse est que les managers ont été davantage sensibilisés aux problèmes de la communication médiatisée par ordinateur, par exemple dans le cadre de séminaires de formation.

Le risque de débordement (9) est également évoqué par des personnels occupants divers postes (J'essaie de faire un traitement dans l'immédiat, sinon, vu le flux, on serait très rapidement débordé). Le courriel peut conduire à ne plus contrôler l'organisation de son temps de travail. C'est souvent la surcharge de travail créée par le courriel qui est mise en avant et très rarement les risques de désorganisation liés aux interruptions, contrairement à ce qui est montré dans certains travaux (Assadi, \& Denis, 2005). L'exemple suivant illustre ce point: Nous avons eu un incident sur un client et en l'espace d'une heure il y a eu six échanges de mails pour ne rien apporter. Ceci parce que les gens sont inquiets et se sentent obligés de se justifier sur ce qu'ils ont apporté dans le traitement de l'incident. Pour moi, c'est une perte de temps, car il faut les lire, y répondre, pour les rassurer. En plus, il faut partir du premier mail et refaire tout l'historique de l'ensemble des messages pour comprendre, car il y a beaucoup de copies et de transferts. D'ailleurs, je n'ai pas encore répondu. Donc, chacun répond à la personne originaire de la demande, mais en mettant des copies à d'autres qui elles-mêmes répondent en mettant encore une autre personne en copie. C'est infernal. Donc j'ai répondu à X qui était à l'origine de la demande, pour le rassurer et lui dire que j'étais au courant et que je prenais en charge. Je n'ai pas répondu aux autres personnes. [L'interviewé lit et commente certains messages]. (Pour moi c'est une perte de temps. Ça gave, car nous avons tellement de choses à faire, avec des mails comme cela c'est une heure de travail en plus).

Le risque de débordement est en lien, de manière logique, avec le sentiment de charge (sept personnes sur neuf qui évoquent ce risque ont un sentiment de charge).

Enfin, la messagerie engendre la diffusion de nombreux messages inutiles, non pertinents pour le destinataire (7) (On en reçoit pas mal, à titre d'information, mais cela n'a pas un grand intérêt). Ce jugement est partagé par des personnels de divers postes.

\subsection{4.- Les limites du courriel}

Deux limites du courriel sont évoquées par les participants. Premièrement, ils soulignent que le courriel n'est pas adapté à la communication de proximité (par exemple, avec son voisin de bureau) (7). Ainsi, le courriel est réservé à la communication avec des personnes distantes physiquement, que ce soit pour limiter la quantité de messages ou pour maintenir des contacts en face à face (Cela va dépendre sur quoi, mais la comptabilité, c'est pareil, je vais passer les voir puisque sinon je ne les verrais jamais non plus).

Deuxièmement, le courriel n'est pas approprié pour traiter des urgences (7) notamment en comparaison du téléphone (Je n'envoie pas de mail quand c'est très urgent je préfère aller voir la personne ou je l'appelle).

Ces deux limites semblent évidentes, car elles sont cohérentes avec les caractéristiques de l'outil (asynchronie et distance). Néanmoins, la partie précédente sur les représentations de l'usage montre que le courriel est souvent utilisé comme moyen de communication synchrone (comme en témoigne la réactivité des participants). Les salariés qui évoquent ces limites manifestent donc leur préférence pour un usage plus « restreint » du courriel.

Ainsi, les propos des participants sont plus orientés vers les désavantages du courriel que vers ses avantages. Cela peut être en partie expliqué par le fait que cet outil est ancré dans les habitudes professionnelles. Ses intérêts semblent aller de soi et ne sont sans doute plus mentionnés. Les mêmes personnes peuvent évoquer des avantages et des jugements négatifs. 
La phase d'analyse suivante est consacrée aux propos décrivant les affects suscités par l'usage du courriel.

\section{3.- Les affects suscités par l'usage du courriel}

Au cours des entretiens, les affects évoqués à propos de l'usage de la messagerie sont plutôt négatifs et parfois intenses (je déteste). Les circonstances d'apparition de ces affects négatifs sont diverses : évaluation des messages reçus, situation engendrée par le courriel (caractère intrusif du courriel), manque de compétences. Plus rarement, le courriel suscite des affects positifs lorsqu'il est associé à une plus grande autonomie (voir Tableau 6). Pour cette dimension de l'expérience, le type de poste occupé ne semble pas être un facteur important.

\begin{tabular}{|l|l|c|}
\hline Type d'émotion & Circonstances d'apparition & $\begin{array}{l}\text { Nbre de } \\
\text { participants }\end{array}$ \\
\hline Colère, irritation, mécontentement & $\begin{array}{l}\text { Messages reçus jugés non } \\
\text { conformes aux règles de savoir- } \\
\text { communiquer }\end{array}$ & 21 \\
\hline Irritation, mécontentement & Situation de (sur)sollicitation & 8 \\
\hline Peur & $\begin{array}{l}\text { Situation de manque de } \\
\text { compétence }\end{array}$ & 4 \\
\hline Contentement & Situation d'autonomie & 2 \\
\hline
\end{tabular}

Tableau 6 : Affects suscités par l'usage du courriel

Table 6: Affects elicited by email usage

\subsection{1.- Colère et évaluation des messages reçus}

La grande majorité des participants évoque des émotions du type colère, irritation ou mécontentement suscitées par les messages reçus, et plus particulièrement par la manière dont ils sont rédigés (21). En fait, les participants considèrent que ces messages violent diverses règles sociales et communicationnelles ou autrement dit les règles de savoircommuniquer (Marcoccia, 1998). Cela concerne les règles de politesse, qui visent à ménager les faces (Brown, \& Levinson, 1987). Par exemple, la politesse implique d'éviter les ordres brutaux ou les remarques désobligeantes. Le cas le plus typique de violation des règles de politesse, dans cette étude, est un message trop directif qui suscite alors des affects négatifs (Je déteste recevoir un mail où on me dit : "Donne-moi ci » ou "Fais-moi cela » (...) Je ne comprends pas qu'on ne commence pas par «Bonjour, est-ce que tu pourrais...»).

Pour d'autres participants, c'est la violation, dans un message, du principe de coopération (Grice, 1979) qui semble être à l'origine d'un affect négatif. Selon ce principe, les individus s'attendent à ce que chacun contribue à l'échange de manière rationnelle et coopérative pour faciliter l'interprétation des énoncés, par exemple en évitant de donner des informations inutiles ${ }^{4}$. Les messages trop longs (comportant trop d'informations) sont un exemple de nonrespect du principe de coopération (Parce que je vois comment je réagis avec les mails qui sont hyper longs. Cela me barbe aussi) tout comme les messages inutiles (non pertinents) ou pas assez clairs (Je me souviens également à propos des premiers modules qu'on a pu faire (...). On avait fait une illustration, et je demandais "c'est quoi le problème ? Si c'est dans la navigation, vous pouvez nous le décrire parce qu'on ne le voit pas sur une copie d'écran, c'est statique...» j'étais assez agacée. Surtout c'étaient des périodes où on avait vraiment la tête dans le guidon en termes de planning, et j'avais écrit une fois à la personne pour lui expliquer qu'on n'avait pas le temps de jouer aux devinettes, que ce serait bien qu'il soit descriptif quand il y a un problème).

Enfin, le non-respect d'autres règles de savoir-communiquer est impliqué dans les affects négatifs des sujets. Par exemple, l'absence de soin dans la rédaction d'un message, en

4 Le principe de coopération renvoie à quatre maximes : quantité (assez mais pas trop d'information), qualité (sincérité), relation (pertinence des propos) et manière (clarté). 
utilisant un style télégraphique, ou en faisant de nombreuses fautes d'orthographe, est mal vécue $^{5}$ (Les mails en style télégraphique cela m'indispose). Par ailleurs, la relation instaurée dans le message peut être perçue comme inappropriée. Par exemple, prendre à témoin un supérieur hiérarchique dans les échanges en le mettant en copie peut sembler s'opposer à l'instauration d'une relation symétrique attendue entre collaborateurs (A chaque fois que l'on m'écrit et que l'on met mon «boss » en copie, cela m'irrite).

L'exemple suivant illustre plusieurs manquements aux règles de savoir-communiquer, le message est inutile et impoli : Là c'est un mail du directeur de l'unité qui envoie un mail aux managers. Chacun des managers répond en remettant tout le monde en copie. Ce n'est pas bien. Il répond pour dire que sur le sujet, il n'est pas d'accord et qu'il souhaite mettre autre chose, c'est bien, mais qu'il réponde à l'intéressé sans arroser tout le monde. Là encore, c'est quelque chose qui m'énerve : on envoie un mail à l'ensemble du comité de direction et on répond: "c'est $\mathrm{OK}$ pour moi » à tout le comité de direction. Il n'y a même pas «bonjour» pas « au revoir», rien.

Ces résultats montrent que les salariés ont une représentation précise de ce qu'est le contrat de communication des échanges professionnels par courriel (politesse, coopération, etc.), mais que ce contrat leur semble souvent non respecté, provoquant ainsi des réactions affectives négatives.

\subsection{2.- Irritation et caractère intrusif du courriel}

Les participants expriment également des émotions de type irritation ou mécontentement, liées à la situation engendrée par le courriel (8). Ainsi, certains participants évoquent la crainte d'être débordés (Les mails c'est trop, c'est un truc qui bouffe (...) Parfois, je pète un câble. Parfois, je me dis "est-ce que je vais y arriver? "Voir aussi l'exemple illustrant le risque de débordement dans 4.2.3) ou simplement le désagrément lié au fait d'être sollicités (Je n'aime pas être polluée de mails donc j'essaie d'y répondre vite). On trouve également des affects négatifs suscités par l'intrusion du courriel dans la sphère privée ( $S$ 'il y a des mails pénibles, que l'on a parfois tard, on ne peut pas pour autant les traiter et cela va pourrir la soirée). Ces résultats montrent ainsi que la réception de courriels est vécue comme une contrainte qui peut peser à différents degrés sur l'activité professionnelle (du désagrément jusqu'au débordement). Parmi les huit individus qui expriment ces affects négatifs, six ont également un sentiment de charge. Pour ceux-là, la quantité de messages semble caractériser la dimension intrusive du courriel.

\subsection{3.- Peur et sentiment de manque de compétence}

L'usage du courriel suscite parfois la peur lorsqu'il est associé au sentiment de mal maîtriser la communication (4), par exemple craindre ne pas être assez poli, de laisser des fautes d'orthographe, ne pas être compris (Je redoute la communication écrite parce qu'elle est difficile et souvent source d'incompréhension, parfois de disputes ou de discorde/L'autre jour j'ai envoyé un mail assez rapide je ne sais plus pour quelle raison, et je me suis dit "ouf, il est parti! J'espère que cela va passer ", j'avais un peu peur et en fait la personne m'a répondu "OK». Après j'ai répondu par un mail un peu plus enveloppé, parce que le mail était vraiment une seule phrase «F. viendra avec toi au rendez-vous »c'est tout. Et donc cela pouvait être mal perçu, et en fait la personne ne l'a pas relevé visiblement, ou bien elle l'a fait, mais elle n'a rien dit. Là-dessus, je l'ai remercié et j'ai été un peu plus... j'ai mis plus de liant). Les quatre personnes qui expriment ces affects négatifs ont aussi évoqué les risques de conflits/malentendus liés au courriel et leur irritation face à certains messages reçus. Ces personnes semblent donc particulièrement sensibles aux difficultés de communication interpersonnelle que peut engendrer le courriel.

5 Les fautes d'orthographe peuvent aussi être vues comme violant le principe de coopération (on ne s'exprime pas de manière claire). Pour cet exemple, ce n'est pas l'interprétation retenue (le problème pour le sujet interviewé n'était pas la difficulté de compréhension). 


\subsection{4.- Affect positif et autonomie}

Deux personnes seulement évoquent des affects positifs lorsqu'elles décrivent leur usage du courriel (contentement). Dans ces cas, les individus associent le courriel à une autonomie dans l'organisation du temps de travail. Pour l'une d'elles, assistante, la messagerie revêt un caractère distractif, car elle permet de se soustraire momentanément à une tâche en cours $(E t$ puis je me sens mieux, c'est comme si je faisais une petite pause (Rire) et je reviens après sur le travail qui me demande de la concentration, donc c'est aussi bien). Pour l'autre personne, le courriel est associé à une liberté dans le choix du moment de traitement des messages. On peut noter que le courriel semble être un outil particulièrement adapté à son activité professionnelle qui nécessite de nombreux échanges avec des consultants externes très mobiles (affectation de personnels dans les formations).

\section{5.- Conclusion et discussion}

L'objectif de cette étude est de décrire l'expérience des usagers du courriel en entreprise et par conséquent d'étudier leurs représentations et leurs affects. Pour cela, nous avons analysé la manière dont les usagers décrivent leur activité de traitement des messages au cours d'un entretien.

Les professionnels interrogés manifestent pour la plupart un engagement permanent dans l'activité de traitement des messages. Le courriel apparait donc, quel que soit le type de poste, comme un outil central dans le travail, qui organise l'activité et qui est utilisé comme dispositif de communication synchrone. Nous avons identifié plusieurs fonctions attribuées au courriel. La fonction principale est celle d'archivage des messages et documents. Les salariés semblent ainsi envisager l'outil essentiellement en position de récepteur et moins souvent d'émetteur. L'usage du courriel est associé au développement de savoir-faire spécifiques dans le domaine de la gestion de l'information (tri, hiérarchisation du traitement) et de la communication (savoir faire un « bon » message).

Les affects évoqués par les participants et les jugements sont, pour l'essentiel, négatifs. Les jugements évoqués sont conformes aux travaux antérieurs. Le sentiment de charge est présent pour de nombreux salariés, le courriel est souvent associé à des risques de débordement et de conflits/malentendus. L'analyse des affects a permis de mettre en évidence des résultats plus originaux comme le fait que l'usage du courriel déclenche souvent de la colère en raison de la violation, dans les messages reçus, des règles de savoircommuniquer (coopération et politesse). D'autres sources d'émotions négatives ont été identifiées : l'irritation due au caractère intrusif et contraignant du courriel, la peur d'être incompétent comme récepteur ou comme rédacteur.

Le type de poste occupé semble peu influencer les affects évoqués. En revanche, concernant les représentations et les jugements, le groupe des managers semble avoir quelques spécificités par rapport aux autres métiers. Presque tous se positionnent sur l'acquisition de compétences associées à l'usage du courriel (la moitié pour signaler leurs lacunes). Ce résultat suggère que ces professionnels accordent davantage d'importance à l'appropriation et à la maîtrise du courriel dans leur métier. Nous faisons l'hypothèse qu'ils perçoivent le «bon » usage des TIC et la qualité de la communication interpersonnelle comme des enjeux importants dans leur profession.

Pour comprendre ce qui dans la situation d'usage du courriel suscite une expérience de valence négative, nous avons croisé les résultats sur les représentations, les jugements et les affects. Au final, trois facteurs principaux permettent d'expliquer le ressenti négatif des usagers : le courriel contribue au sentiment de contrôler plus difficilement l'activité de travail, les échanges par courriel sont perçus comme violant les règles de savoircommuniquer et le courriel peut parfois susciter un sentiment de manque de compétence.

Premièrement, l'usage du courriel peut s'accompagner d'un sentiment de diminution $d u$ 
contrôle sur l'activité de travail. Les participants expriment un sentiment de charge (quantité de messages reçus). La surcharge (ou débordement) est perçue comme un risque et suscite des craintes. Par ailleurs, les représentations des participants concernant leur usage du courriel suggèrent qu'il est source de travail intense (fort engagement, réactivité). On peut donc faire l'hypothèse que le surcroit d'activité et de demande psychologique (Karasek, Brisson, Kawakami, Houtman, Bongers, \& Amick, 1998) conduit les salariés à percevoir la situation comme moins contrôlable, et constitue ainsi une source d'affects négatifs. En d'autres termes, les contraintes générées par le courriel pourraient conduire les salariés à avoir le sentiment de moins facilement contrôler les évènements (que le contrôle soit effectif ou non). De plus, recevoir un message est toujours une contrainte dans la mesure où il crée des obligations réciproques : devoir de réponse et devoir de réactivité (Akrich, Meadel, \& Paravel, 2000) en raison des engagements pris avec les différents interlocuteurs (historique des relations...). De la même manière, Licoppe (2009) considère qu'un message reçu implique une charge morale, car il appelle une réponse, notamment en raison de l'organisation séquentielle des conversations. Les participants témoignent de cette contrainte en évoquant le désagrément lié au fait d'être sollicités. Par ailleurs, comme le message est persistant, la contrainte est toujours visible jusqu'à ce que le message soit traité (réponse, suppression...). Ainsi, l'usage du courriel peut conduire à un sentiment de perte de contrôle dans le sens où les messages arrivants freinent ou modifient le but en cours (ils ajoutent de nouvelles contraintes à la situation).

Deuxièmement, les échanges par courriel sont perçus comme violant les règles de savoircommuniquer. En effet, presque tous les participants ont évoqué des réactions affectives négatives (notamment l'irritation) suscitées par les messages reçus. Ces messages ne respectent pas, selon les participants, un certain nombre de règles communicationnelles (être concis, poli, etc.). Par ailleurs, le risque le plus souvent associé au courriel est celui de conflit et/ou d'incompréhension dans les échanges. Comment expliquer ce sentiment partagé par tous les usagers que leurs collaborateurs violent les règles de savoir-communiquer dans leurs messages ? En fait, le problème, selon nous, n'est pas que, de manière générale, le courriel conduit à transgresser les règles de la communication. Le problème est que les usagers ne partagent pas la même représentation du contrat de communication des échanges par courriel. Cette difficulté à partager des règles communes est liée au fait que les règles de la communication par courriel ne sont pas fixes et sont complexes. En effet, le courriel est une forme de CMO hybride qui combine les caractéristiques de la communication écrite (notamment les courriers professionnels) et celles de la conversation (notamment la conversation téléphonique) (Baron, 2000; Marcoccia, 2000). Par ailleurs, les formes du courriel peuvent se transformer avec l'évolution des médias de communication. Par exemple, le fait de répondre aux courriels sur smartphone conduit à rédiger des messages plus courts (Karlson, Meyers, Jacobs, Johns, \& Kane, 2009).

Troisièmement, le courriel est parfois associé à un sentiment de manque de compétence dans l'usage de l'outil. L'appropriation de la messagerie, comme pour d'autres outils, implique le développement d'un savoir-faire. On pouvait s'attendre à ce que l'expérience assez ancienne de la messagerie par les participants les ait amenés à développer ces savoir-faire. Pourtant, certains expriment le sentiment de ne pas maitriser la messagerie du point de vue de sa fonction d'archivage (trier, traiter efficacement les messages) et/ou de sa fonction de communication (rédiger des messages de manière appropriée). La crainte de ne pas utiliser la messagerie de manière appropriée peut être vue comme une source de travail empêché, contrarié (Clot, 1999). Dans l'entreprise étudiée (comme dans beaucoup), la messagerie est un outil central dans le travail ; elle sert tout autant aux échanges avec le client qu'à la collaboration entre collègues. Par conséquent, ne pas maîtriser la messagerie peut être perçu comme un obstacle pour être efficace, pour produire un travail de qualité. Le caractère contraignant du courriel, évoqué précédemment, peut également être vu comme une source de travail empêché. Les messages arrivants perturbent l'activité prévue, risquent de la freiner 
ou de modifier le but en cours. Ainsi, la prise en charge de la formation des salariés au courriel par les entreprises et la mise en œuvre d'actions favorisant une réflexion sur l'usage du courriel par le collectif de travail, semblent nécessaires.

\section{6.- Limites et perspectives}

Une des limites de cette étude est le recours exclusif aux méthodes d'entretiens. En effet, les entretiens ne permettent d'accéder qu'à une partie seulement de l'activité. L'observation des usages n'a pas été possible dans cette étude, mais elle aurait permis de renseigner de manière plus fine les tâches en lien avec la messagerie, les stratégies d'usage spécifiques des outils de communication et les affects (manifestés, mais non verbalisés). Ces aspects de l'activité auraient été utiles pour comprendre l'expérience des usagers. De plus, les entretiens que nous avons réalisés ne semblent pas avoir fonctionné de manière satisfaisante. En effet, les participants font insuffisamment référence à des cas précis et spécifiés. Cela est lié au fait que nous avons voulu mener plusieurs objectifs dans l'entretien (entretien sur les pratiques en général, mais aussi sur des exemples précis) et que nous disposions d'un temps limité (une heure). Sans doute que le fait d'interroger les participants au préalable sur leur usage en général ne crée pas des conditions favorables pour qu'ils sortent ensuite d'un discours général. De plus, nous n'avons sans doute pas consacré suffisamment de temps dans l'entretien sur les situations précises (par exemple, pour permettre aux participants d'être en évocation dans la phase d'explicitation).

Une autre limite de cette étude est l'échantillonnage des participants. Notre objectif était d'interroger des salariés aux profils variés pour obtenir des données sur des types de postes habituellement peu étudiés. Cet objectif a été rempli, mais la variété des postes occupés limite la portée des résultats. En effet, nous avions souvent un seul représentant de chaque métier sauf pour les managers ( 9 personnes) et les consultants-formateurs ( 5 personnes). Dans l'ensemble, le métier et le niveau hiérarchique n'ont pas semblé jouer un rôle déterminant. Le métier de manager est le seul pour lequel nous avons pu identifier quelques spécificités. Il serait donc intéressant de confirmer ces résultats en opérant des comparaisons sur des échantillons plus importants.

Cette étude permet de contribuer à la compréhension de la qualité de l'expérience utilisateur avec des objets familiers (situations d'usage sur le long terme). En effet, elle suggère des pistes pour identifier les facteurs en jeu dans le développement d'une expérience utilisateur positive et dans l'acceptabilité d'une technologie. Notamment, le rôle de la technologie dans le contrôle de l'activité (permet-elle ou au contraire limite-t-elle le contrôle ?) semble être un facteur central au-delà de la situation d'usage du courriel. Par exemple, Cahour (2010) observe que l'inconfort dans les situations d'usage (systèmes de communication, conduite automobile) est souvent lié à une perte de contrôle de l'action habituelle.

De plus, dans le cas du courriel, nous avons pu observer que les participants se mettaient essentiellement en position de récepteur et moins souvent d'émetteur alors que l'entretien portait également sur les messages envoyés. Cette position est sans doute liée au fait que l'usage du courriel se caractérise, en termes d'activité et de temps consacré, essentiellement par la réception (attente d'un message, lecture, archivage). Utiliser le courriel serait avant tout perçu comme recevoir des messages et les traiter. La prégnance de cette position de récepteur semble influencer l'expérience utilisateur dans le sens d'un sentiment de contrôle plus faible. Il serait donc intéressant de vérifier que cette position de récepteur est également prégnante avec d'autres technologies de communication comme le téléphone ou bien si cela est spécifique à la situation d'usage du courriel en entreprise.

Enfin, cette étude montre que l'expérience utilisateur est influencée par les usages du dispositif par le collectif. Par exemple, des usages qui ne sont pas homogènes dans le cas de la rédaction des messages (usage de règles de savoir-communiquer différentes) suscitent une 
expérience négative du courriel. Dans les modèles évoqués précédemment (Mahlke, \& Thüring, 2007), l'UX est plutôt conçue comme le résultat d'une interaction entre une personne et un dispositif même si des variables sociales sont censées pouvoir influencer l'expérience. Cette position apparaît insuffisante pour rendre compte de la construction de l'expérience utilisateur pour des technologies familières. Ainsi, l'usage d'une technologie par le collectif (groupe de travail, consommateurs, etc.) est une dimension importante à développer dans les modèles de l'UX.

\section{BIBLIOGRAPHIE}

Abric, J.C. (1994). Pratiques sociales, représentations sociales, In J.C. Abric (Ed.). Pratiques sociales et représentations (217-238). Paris: PUF.

Akrich, M., Meadel, C., \& Paravel, V. (2000). Le temps du mail : écrit instantané ou oral médiat. Sociologie et sociétés, 32 (2), 154-171.

Assadi, H., \& Denis, J. (2005). Les usages de l'e-mail en entreprise : efficacité dans le travail ou surcharge informationnelle? In E. Kessous \& J.M. Metzger (Eds.). Le travail avec les technologies de l'information (135-155). Paris: Hermes.

Bagozzi, R., \& Lee, K.-H. (1999). Consumer resistance to, and acceptance of, innovations. Advances in Consumer Research, 26, 218-225.

Barcenilla, J., \& Bastien, C. (2009), L'acceptabilité des nouvelles technologies : quelles relations avec l'ergonomie, l'utilisabilité et l'expérience utilisateur? Le Travail humain, 72, 311-331.

Baron, N.S. (2000). Alphabet to Email, How written English evolved and where it's heading. London: Routledge.

Besseyre des Horts, C.-H. (2008). L'entreprise mobile: Comprendre l'impact des nouvelles technologies. Paris: Paerson Education France.

Brown, P., \& Levinson, S. (1987). Politeness: Some universals in language usage. Cambridge: Cambridge University Press.

Cahour, B. (2010). Emotion, affects et confort comme nouveaux déterminants de l'activité et de l'usage. In G.Valléry, M.-C. Leport, \& M. Zouinar (Eds). Ergonomie, conception de produits et services médiatisés (pp. 273-306). Paris: PUF.

Cahour, B., Brassac, C., Vermersch, P., Bouraoui, J.-L., Pachoud, B., \& Salembier, P. (2007). Étude de l'expérience du sujet pour l'évaluation de nouvelles technologies : l'exemple d'une communication médiée. Revue d'anthropologie des connaissances, 1(1), 85-120.

Chaudhuri, A., \& Holbrook, M. (2001). The Chain of Effects from Brand Trust and Brand Affect to Brand Performance: The Role of Brand Loyalty. Journal of Marketing, 65(2), 81-93.

Chitturi, R., Raghunathan, R., \& Mahajan, V. (2008). Delight by design: the role of hedonic versus utilitarian benefits. Journal of Marketing, 72, 48-63.

Clot, Y. (1999). La fonction psychologique du travail. Paris: PUF.

Cosnier, J. (1994). Psychologie des émotions et des sentiments. Paris: Retz.

Czerwinski, M., Horvitz, E., \& Wilhite, S. (2004). A diary study of task switching and interruptions, In Human Factors in Computing Systems: Proceedings of CHI'04. pp. 175-182. New York: ACM Press.

Dactachary, C. (2009). La dispersion au travail. Toulouse: Octarès Édition.

Datchary, C., \& Licoppe, C. (2007). La multi-activité et ses appuis : l'exemple de la "présence obstinée" des messages dans l'environnement de travail. Activités, 4 (1), 4-29.

Desmet, P. M., \& Hekkert, P. (2007). Framework of product experience. International Journal of Design, 1(1), 57-66.

Ducheneaut, N., \& Bellotti V. (2001). Email as a habit: An exploration of embedded personal information management. Interactions, 8 (5), 30-38.

Ducheneaut, N., \& Watts, L. (2005). In Search of Coherence: A Review of E-Mail Research. HumanComputer Interaction, 20, 11-48.

Feldman-Barrett, L., Mesquita, B.,Ochsner, K., \& Gross, J. (2007). The Experience of Emotion. The Annual Review of Psychology, 58, 373-403. 
Février, F., Gauducheau, N., Jamet, E., Rouxel, G., \& Salembier, P. (2011). La prise en compte des affects dans le domaine des interactions homme-machine : quels modèles, quelles méthodes, quels bénéfices? Le Travail Humain, 74, 183-192.

Forgas, J. (2007). When sad is better than happy: Negative affect can improve the quality and effectiveness of persuasive messages and social influence strategies. Journal of experimental social psychology, 43(4), 513-528.

Frijda, N.H. (1986). The emotions. Cambridge: Cambridge University Press.

Garrett, J. (2006). Customer loyalty and the elements of user experience. Design management review, $17(1), 35-39$.

Grice, H.P. (1979). Logique et conversation. Communications, 30, 57-72.

Hair, M., Renaud, K. V., \& Ramsay, J. (2007). The influence of self-esteem and locus of control on perceived email-related stress. Computers in Human Behavior, 23(6), 2791-2803.

Hassenzahl, M. (2004). The Interplay of Beauty, Goodness, and Usability in Interactive Products. Human-Computer Interaction, XIX, 319-349.

Hassenzahl, M. (2008). User Experience (UX): Towards an experiential perspective on product quality. In Actes de la conférence IHM 2008 (pp. 11-15). New York: ACM Press.

Hassenzahl, M, \& Tractinsky, N. (2006). User Experience- a research agenda. Behaviour and Information Technology, 25(2), 91-97.

Isaac, H., Campoy, E., \& Kalika, M. (2007). Surcharge informationnelle, urgence et TIC. L'effet temporel des technologies de l'information. Revue management et avenir, 3(13), 149-168.

Isen, A. (2000). Positive affect and decision making. In M. Lewis, M. \& J. Haviland-Jones, J. (Eds.), Handbook of emotions (pp. 417-435). NY: The Gilford Press.

Jackson, T., Dawson, R. J., \& Wilson, D. (2001). The cost of email interruption. Journal of Systems and Information Technology, 5(1), 81-92.

Jordan, P. (2000). Designing pleasurable products, London: Taylor \& Francis.

Karapanos, E., Zimmerman, J., Forlizzi, J., \& Martens, J.-B. (2009). User Experience over time: An initial Framework. In Proceedings of CHI 2009 (pp. 729-738). New York: ACM.

Karasek, R., Brisson, C., Kawakami, N., Houtman, I., Bongers, P., \& Amick, B. (1998). The Job Content Questionnaire (JCQ): an instrument for internationally comparative assessments of psychosocial job characteristics. Journal of Occupational Health Psychology, 3(4), 322-355.

Karlson, A.K., Meyers, B.R., Jacobs, A., Johns, P., \& Kane, S.K. (2009). Working overtime: Patterns of smartphone and PC usage in the day of an information worker. In Proceedings of the Seventh International Conference on Pervasive Computing (Pervasive '09) (pp. 398-405). Berlin: Springer.

Kujula, S., Roto, V., Väänänen-Vainio-Mattila, K., Karapanos, E., \& Sinnelä, A. (2011). UX Curve: A method for evaluating long-term user experience. Interacting with Computers, 3(5), 473-483.

Lahlou, S. (2000). Attracteurs cognitifs et travail de bureau. Intellectica. 1(30), 75-113.

Law, E., Roto, V., Hassenzahl, M., Vermeeren A., \& Kort, J. (2009). Understanding, Scoping and Defining User Experience: A Survey Approach. In Proceedings of Human Factors in Computing Systems conference, CHI'09 (pp. 719-728). New York: ACM.

Lerner, J.S., \& Keltner, D. (2000). Beyond valence: Toward a model of emotion specific influences on judgment and choise. Cognition and Emotion, 14(4), 473-493.

Licoppe, C. (2009). Pragmatique des interruptions et des notifications et ethnographie des situations de travail. In B. Cahour, F. Anceaux, \& A. Giboin (Eds.). Actes de la 9ème conférence Epique (pp. 240-244).

Light, A. (2006). Adding method to meaning: A technique for exploring peoples' experience with technology. Behaviour and Information Technology, 25(2), 175-187.

Mahlke, S., \& Thüring, M. (2007). Studying Antecedents of Emotional Experiences in Interactive Contexts. In Proceedings of CHI 2007 (pp. 915-918). New York: ACM.

Mallein, P., \& Toussaint, Y. (1994). L'intégration sociale des technologies d'information et de communication. Une sociologie des usages. Technologies de l'information et société, 4, 315-335.

Mano, R. S., \& Mesch, G. S. (2010). E-mail characteristics, work performance and distress. Computers in Human Behavior, 26(1), 61-69. 
McCarthy, J., \& Wright, P. (2004). Technology as Experience. Cambridge Mass: MIT Press.

Marcoccia, M. (1998). La Normalisation des comportements communicatifs sur Internet : étude sociopragmatique de la Netiquette. In N. Guégen, \& L. Tobin (Eds.). Communication, société et internet (pp. 15-32). Paris: L'Harmattan.

Marcoccia, M. (2000). Les smileys : une représentation iconique des émotions dans la communication médiatisée par ordinateur. In C. Plantin, M. Doury, \& V. Traverso (Eds.). Les émotions dans les interactions (pp. 249-263). Lyon: ARCI. Presses universitaires de Lyon.

Mollo, V., \& Falzon, P. (2004). Auto- and allo-confrontation as tools for reflective activities. Applied ergonomics, 35, 531-540.

Norman, D. A. (2004). Emotional Design: Why we Love (or Hate) Everyday Things. New York: Basic Books.

O'Kane, P., \& Hargie, O. (2007). Intentional and unintentional consequences of substituting face-toface interaction with e-mail: An employee-based perspective. Interacting with computers, 19, 2031.

Paillé, P., \& Mucchielli, A. (2003). L'analyse qualitative en sciences humaines et sociales. Paris: Armand Colin.

Russell, E., Purvis, L. M., \& Banks, A. (2007). Describing the strategies used for dealing with email interruptions according to different situational parameters. Computers in Human Behavior, 23(4), 1820-1837.

Scherer, K.R. (2001). Appraisal considered as a process of multi-level sequential checking. In K. R. Scherer, A. Schorr and T. Johnstone (Eds.). Appraisal processes in emotion: Theory, Methods, Research.pp. 92-120. New York and Oxford: Oxford University Press.

Scherer, K.R. (2005). What are emotions? And how should they be measured? Social Science Information, 44(4), 695-729.

Sproull, L., \& Kiesler, S. (1986). Reducing social context cues: Electronic mail in organizational communication. Management Science, 32(11), 1492-1512.

Theureau, J. (2000). Anthropologie cognitive et analyse des compétences. In J-M Barbier (Ed.), L'analyse de la singularité de l'action (pp. 171-211-. Paris: PUF.

Vermersch, P. (1991). L'entretien d'explicitation. Les Cahiers de Beaumont, 52 bis-53, 63-70.

Whittaker, S., \& Sidner, C. (1997). Email overload: Exploring personal information management of email. In Kiesler, S. (Ed.). Culture of the Internet (pp. 277-295). New Jersey: Lawrence Erlbaum Associates.

Wood, S., \& Moreau, P. (2006). From Fear to Loathing? How Emotion Influences the Evaluation and Early Use of Innovations. Journal of Marketing, 70, 44-57.

\section{RESUME}

L'objectif de cet article est de décrire l'expérience qu'ont les individus de l'usage du courriel en situation de travail. Vingt-cinq entretiens inspirés des techniques d'explicitation et d'autoconfrontation ont été réalisés auprès d'employés d'une entreprise de formation. Les entretiens ont permis de mettre au jour trois aspects de l'expérience des usagers: les représentations de leur activité de traitement des messages, leurs jugements sur le courriel (avantages, désavantages) et les affects suscités par son usage. L'analyse montre que l'expérience négative des participants est liée à trois facettes de la situation d'usage du courriel. Tout d'abord, l'usage du courriel s'accompagne souvent du sentiment de contrôler plus difficilement l'activité de travail. Ensuite, les échanges par courriel sont perçus comme violant les règles de savoir-communiquer. Enfin, les participants ont parfois le sentiment de manquer de compétence dans l'usage de l'outil. 


\section{MOTS CLES}

expérience utilisateur, activité professionnelle, courriel, affects, représentations

\section{REFERENCEMENT}

Gauducheau, N. (2012). L'expérience du courriel en situation professionnelle: représentations de l'activité, jugements et affects. Activités, 9(2), 88-111, http://www.activites.org/v9n2/v9n2.pdf

Article soumis le 4 novembre 2011, accepté pour publication le 30 mai 2012 